\title{
Ranking and prioritization with multiple indicators in digital governance and surveillance hotspot geoinformatics: a preface
}

\author{
G. P. Patil
}

Received: 25 October 2010 / Revised: 27 October 2010 / Published online: 9 December 2010

(C) Springer Science+Business Media, LLC 2010

\section{Introduction and invitation}

This article is prepared in the spirit of inviting the attention of the readership to some of the initiatives of the author and his collaborators that have presently culminated into a novel and innovative initiative for digital governance and hotspot geoinformatics around the world. Geoinformatics of geospatial and spatio-temporal hotspot detection and prioritization is a critical need for the twenty-first century. A declared need is around for statistical geoinformatics and software infrastructure development. A hotspot can mean an unusual phenomenon, anomaly, aberration, outbreak, elevated cluster, critical area. The declared need may be for monitoring, etiology, early warning or sustainable management. The responsible factors may be natural, accidental or intentional. The seven year NSF Digital Government Research Program project has been instrumental to conceptualize hotspot geoinformatics partnership among several interested cross-disciplinary scientists in academia, agencies, and communities around the world. Our efforts are driven by a wide variety of case studies involving a wide variety of critical societal issues.

You are invited to participate in ongoing workshop series around the world in a manner most productive for your purpose and publications. You will have the opportunity to strengthen, advance, and accelerate your in-house research work plan and developmental effort involving novel geoinformatics and innovative hotspot dynamics

This material is based upon work supported by the US National Sicience Foudnation under Grant No. 0307010. Any opinions, findings, and conclusions or recommendations expressed in this material are those of the author(s) and do not necessarily reflect the views of the agencies.

G. P. Patil $(\varangle)$

Department of Statistics, Center for Statistical Ecology and Environmental Statistics, The Pennsylvania State University, University Park, PA 16802, USA

e-mail: gpp@stat.psu.edu 
with capability for early warning and sustainable management. It will be a pleasure to communicate, interact and publish. See the website: http://www.stat.psu.edu/hotspots/ pdfs/OverallInfo_shortcourseandworkshops.pdf and also the twenty-five referenced websites in it. Also see Patil (2010).

\section{Motivation}

The motivation may best be shared from the backcover page material prepared for the 400 page monograph on, "ranking and prioritization for multi-indicator systems: introduction to partial order applications", by Brüggemann and Patil (2010a) (in press).

Ranking issues are found everywhere! For example, bank houses, universities, towns, watersheds etc. are ranked. But also assessment of students in one discipline is a ranking. This last example is trivial, because we have only one criterion, namely the quality of the student in that discipline. In the other cases ranking can be a very hard job. Why? There is often no measure. How do we measure towns with respect to living quality? How do we measure the hazard exerted by chemicals? No chemical has its intrinsic identity card where its hazard can be identified.

Thus multi-indicator systems come into play. We gather indicators which help to characterize the items of interest for ranking. A lot of work! Measurement of indicators, selecting indicators, testing indicators. And we arrive at a multi-indicator system. Are we done?

No! We have gathered useful information for ranking. However, we do not know how to derive ranking from the multitude of valuable information. In a popular approach, the indicator values are weight-averaged. The resulting weighted averages are used to obtain the ranking.

Are we done?

No! Where is all the detailed information and the amount of work, which was put into every single indicator? It has disappeared as it does, when an averaging process is applied.

Can we offer an alternative? Yes, we can. We offer the mathematical tool of partial order as a tool to get insight into the process, starting with the multi-indicator system and finishing up with ranking. Application of partial order involving multi-indicator systems is in its initial phases and is advancing with more and more tools.

This special issue provides a timely introduction to the partial order theory and its techniques with worked out illustrations and applications to a variety of live case studies.

\section{Introducing the five invited papers of this special issue}

The first paper by Brüggemann and Patil (2010b) deals with multicriteria prioritization and partial order in environmental sciences. The complexity of the present data-centric world finds its expression in the increasing number of multi-indicator systems. This has led to the development of multicriteria ranking systems based on partial orders. Order theory is a main pillar of structural mathematics. Partial orders help to reveal why an object of interest holds a certain ranking position and how much it is subject 
to change if a composite indicator is upgraded. Order theory helps to derive linear or weak orders without indicator weighting schemes. Hence, rankings obtained from decision support systems (DSS) which depend on many parameters beyond the data matrix can be checked and discrepancies can lead to examine the parameters of the DSS. Order theory helps discover association and implication structures derived from formal concept lattices. Association and implication networks among the attributes of the data matrix allow more insights into multi-indicator systems and lead to new hypotheses and motivate further research. Some new and innovative concepts, like separated subsets, antagonistic indicators, ranking stability fields are rendered. Separated subsets are the typical outcome of a partial order analysis; their identification leads to antagonistic indicators, which are responsible for the separatedness of object's subsets. Numerical aggregation can be performed step-by-step and the question which values of a weight lead to an order inversion is of high interest. The concept of stability fields is one possible answer, discussed in this paper. After an outline of partial order theory some more specific theoretical results are shown, then we discuss the role of composite indicators in the light of partial order and give some examples of interesting applications of partial order. Finally examples are selected from real life case studies of watersheds, environmental performance evaluations, child well being, geographic and administrative regions and more.

The second paper by Myers and Patil (2010) deals with preliminary prioritization based on Partial order theory and R software for compositional complexes in landscape ecology, with applications to restoration, remediation and enhancement. The present purpose is to provide convenient computational capability and visualizations for preliminary partial or progressive prioritization based largely on concepts of partial order theory and implemented in $\mathbf{R}$ software as illustrated in a context of conservation and sustainable stewardship across landscapes with ecosystem services as a complex multidimensional domain that must be placed in public and private perspective in pursuit of multi-resource management. Practical perspective is promoted by graphic visualization with local partial order modeling (LPOM) methods for screening of settings and scenarios involving interactions of ecosystem elements as evidenced by environmental indicators. ORDIT ordering and precedence plots arise from ascribed advantage as an outcome of a rating regime. Representative ranks constitute criteria drawn from the rank distribution for the case in question. Distal data are determined with regard to remediation and retention. Median mismatches reflect interplay of indicators appearing as isolated instances in plotting patterns. A suggested strategy to circumvent computational constraints is partitioning the pool of cases into collectives by clustering, pursuing classes of partitions, and then prioritizing in particular partitions. When prime prospects have been obtained, detailed determinations can be done with partial ordering procedures involving Hasse diagrams and similarly complex constructs that are difficult to apply with many cases and/or indicator criteria.

The third paper by Newlin and Patil (2010) with application of partial order to bridge engineering, stream channel assessment, and infrastructure management. Many environmental systems and infrastructure systems are monitored using a set of indicator values that assess several aspects of site condition. The values of different indicators often convey different comparative messages for the study sites. The comparability between sites is analyzed within the theory of partially-ordered sets and visualized 
with Hasse diagrams. This paper demonstrates that properties of partially ordered sets and the conversion of indicator scores into a rank matrix provide a manager or decision-maker with valuable information that would be lost if the monitored indicator data were aggregated only into a single index. Stream stability assessment data from 49 bridge crossing sites in the United States are used to demonstrate these techniques in prioritization and identification of restoration and maintenance needs of stream channels at bridge crossings.

The fourth paper by Yang and McManus (2010) deals with infant mortality and social environment in Georgia : an application of hotspot detection and prioritization. Recent years have witnessed the growth of new information technologies and their applications to various disciplines. The goal of this paper is to demonstrate how the two innovative methods, upper level set scan (ULS) hotspot detection and the multicriterion prioritization scheme, facilitate population health and break new ground in public health surveillance. It is believed that the social environment (i.e. social conditions and social capital) is one of the determinants of human health. Using infant health data and 10 additional indicators of social environment in the 159 counties of Georgia, ULS identified 52 counties that are in double jeopardy (high infant mortality and a high rate of low infant birth weight). The multicriterion ranking scheme suggested that there was no conspicuous spatial cluster of ranking orders, which improved the traditional decision making by visual geographic cluster. Both hotspot detection and ranking methods provided an empirical basis for re-allocating limited resources and several policy implications could be drawn from these analytic results.

The fifth paper by Pecci et al. (2010) deals with biodiversity protection funding preference: a case study of hotspot geoinformatics and digital governance for the map of Italian Nature in the presence of multiple indicators of ecological value, ecological sensitivity and anthropic pressure for the Oltrepò Pavese and Ligurian-Emilian Apennine study area in Italy. The environmental decision-maker is aware of the increasing difficulties in finding sufficient financial resources for nature conservation. So he must focus his attention on ecological situations that more than the others merit considering and defending because of elevated value but also because of risk for their intrinsic characteristics and for human pressure acting on them.

Usually an ecological scientist focuses his attention on the natural patches of the landscape, analyzing their peculiar ecological traits forgetting that, even if we want to protect some environmental critical situations, this can be done only moving to the administrative partition of the territory since the central and local environmental stakeholders have primary interest in providing funds to those involved in those critical situations.

The present work shows a methodological approach, consisting of a set of statistical and geoinformational tools, considering both ecological and socio-demographical indicators. The goal is not simply to give some general guidelines for environmental policies to the involved stakeholders but focuses more on finding out which administrative local partitions in a study area are more worthy to receive urgently the priority funds for biodiversity protection to face critical environmental situations often due to a combination of intrinsic ecological parameters and external human pressure ones. Obtaining results that cover $5 \%$ of the Communes involved in the area seems to be a realistic result that a decision-maker can support and fund. 
Methodologically and geospatial data analytically, the investigation offers interesting challenges for surveillance geoinformatics of hotspot detection and prioritization, because of the presence of multiple hotspots and multiple sets of multiple indicators.

\section{References}

Brüggemann R, Patil GP (2010a) Ranking and prioritization for multi-indicator systems: introduction for partial order applications. Springer, New York (in Press)

Brüggemann R, Patil GP (2010b) Multicriteria prioritization and partial order in environmental sciences. (this issue)

Myers WL, Patil GP (2010). Partial order and rank range runs for compositional complexes in landscape ecology and image analysis, with applications to restoration, remediation, and enhancement. Environ Ecol Stat (this issue)

Newlin JT, Patil GP (2010) Application of partial order to bridge engineering, stream channel assessment, and infra structure management. Environ Ecol Stat (this issue)

Patil GP (2010) Digital governance, hotspot geoinformatics, and sustainable development: a preface. Environ Ecol Stat 17(2):132-147

Pecci A, Patil GP, Rossi O, Rossi P (2010) Biodiversity protection funding preference: a case study of hotspot geoinformatics and digital governance for the map of Italian nature in the presence of multiple indicators of ecological value, ecological sensitivity and anthropic pressure for the oltrepò pavese and Ligurian-Emilian Apennine study area in Italy (this issue)

Yang TC, McManus B (2010) Infant mortality and social environment in georgia: an application of hotspot detection and prioritization (this volume)

\section{Author Biography}

G. P. Patil is a distinguished professor of mathematical and environmental statistics emeritus and director of the Center for Statistical Ecology and Environmental Statistics in the Department of Statistics at the Pennsylvania State University. He is founding Editor-in-Chief of the International Journal: Environmental and Ecological Statistics. He is Series Editor of the Monographs on Environmental and Ecological Statistics. He has been a Fellow of ASA, IMS, and AAAS. He has received the distinguished statistical ecological award of the International Association for Ecology and the distinguished achievement medal of the American Statistical Association. He is just about finishing as the PI a large cross-disciplinary seven year NSF project on digital governance and surveillance hotspot Geoinformatics for monitoring, etiology, early warning, and sustainable development. This has involved upper level set scan based geospatial and spatiotemporal hotspot detection and partial order theory based multi-criteria ranking and prioritization with several national and international live case studies. 